\title{
Intent For Mitigation
}

National Cancer Institute

\section{Source}

National Cancer Institute. Intent For Mitigation. NCI Thesaurus. Code C54416.

The use of a particular medical device, medication or treatment to help minimize the effects of a disease or condition. 